\section{Rotation judgments and depth judgments: Separate or dependent processes?}

\author{
J. TIMOTHY PETERSIK \\ Southeast Missouri State University \\ Cape Girardeau, Missouri 63701
}

In his 1976 review of the factors yielding the perception of depth in kinetic displays, Braunstein offers the suggestion that "separate processes are involved in depth and direction judgments" (p. 89) of multielement rotation displays. His conclusion seems to be primarily based upon the results of two studies in which depth discrimination and coherence discrimination were different functions of perspective magnitude and element numerosity (Braunstein, 1962) and in which depth discrimination and rotation-direction discrimination were different functions of perspective magnitude. Although Braunstein's results are suggestive, they do not necessarily warrant the "separate processes" conclusion. This is true for the following reasons: (1) In neither study was the magnitude of the depth sensation produced by a given display directly estimated. Rather, subjects made forcedchoice judgments (i.e., either "more depth, less depth" or "three-dimensional, not three-dimensional"). (2) In the first study (Braunstein, 1962), the number of elements varied between only one and six dots per display. Given that such small variations in element numerosity may not reveal interesting changes in the perceived depth function, Braunstein's conclusion rests essentially on the observation that depth discrimination and rotation discrimination are different functions of perspective magnitude. In the present paper, a more detailed examination of the relationship between depth sensations and rotation-discrimination is made, and the conclusion is drawn that no simple statement relating the processes underlying depth and rotation sensations can be advanced at this time. Furthermore, we suggest that the term "functional dissociation" be used to describe the candidate relationship between depth and rotation sensations. ${ }^{1}$ In the course of two previously reported studies (Petersik, in press), subjects viewed computer-animated simulations of threedimensional (3D) spheres rotating around the vertical axis in depth. The displays were discrete rather than continuous, and therefore the resulting sensations of motion were stroboscopic. During the course of the two experiments, several independent variables were manipulated: The most important of these were frame duration, interstimulus interval (ISI), sphere size,

Requests for reprints should be addressed to: J. Timothy Petersik, Department of Psychology, Southeast Missouri State University, Cape Girardeau, Missouri 63701. simulated projection distance (i.e., perspective), dot numerosity, and number of frames per 360-deg rotation. A total of 12 subjects viewed these displays, and on each trial a subject both rated the depth (0-6) of the simulated sphere and identified its apparent rotation direction. After the primary effects of the independent variables were analyzed, we specifically examined the relationship between the functions relating perceived depth and rotation discrimination to the independent variables.

\section{The Assumption of Functional Dissociation}

(1) One source of evidence suggesting that depth and rotation discrimination might be functionally dissociated was derived from the curves that related the dependent measures to stimulus onset asynchrony (SOA, 20-700 msec). These curves are shown in Petersik's (in press) Figures 1 and 2. The curve relating the percentage of correct rotation judgments to SOA gives the appearance of an inverted $U$ with a steady decline at SOAs less than the peak value of $210 \mathrm{msec}$. On the other hand, the curve relating depth judgments to SOA does not show a decline at the shorter SOAs. The depth judgments at SOAs of $210 \mathrm{msec}$ and less are all within the same range, between 3.5 and 4.0. Thus, the curves relating the two judgments to SOA appear to reflect the operation of underlying processes with different temporal response properties. That is, the temporal response function for rotation discrimination has a relatively narrow suprathreshold temporal bandwidth, with a half-width at half-amplitude of 10.0-12.5 Hz. The function shows sharp declines at temporal frequencies both above and below the optimum frequency of $4.76 \mathrm{~Hz}$. On the other hand, the temporal response function for depth ratings is broad, with a cutoff only for low temporal frequencies. Given this information about the temporal response characteristics of the present two dependent judgments, it might be possible to model the perceptual processes underlying rotation-direction judgments after transient visual mechanisms, which have a peak temporal sensitivity in the neighborhood of $3-5 \mathrm{~Hz}$. Similarly, it might be possible to model the present depth responses with sustained visual mechanisms, which may require several stimulations throughout a relatively long integration period in order to produce the best response (cf. Pantle, Note 1.) However, the evidence suggesting relationships between transient mechanisms and the present perception of depth is only suggestive at this point. More data relevant to these comparisons need to be collected before the appropriate models can be constructed.

The difference between the curves in Petersik's (in press) Figures 1 and 2 does suggest, however, that 
different perceptual mechanisms underlie rotation and depth judgments in the rotating sphere simulation. This suggestion (and even the hypothetical relationship to sustained-i.e., pattern - and transienti.e., flicker-mechanisms) is consistent with Richards' $(1975$, p. 378$)$ thesis that there are essentially two perceptual systems involved in visual space perception.

(2) In addition, the figures relating rotation discrimination and perceived depth to projection distance are quite different in appearance, despite the fact that they both reflect significant main effects of perspective. The figure relating percentage of correct rotation-direction judgments to projection-point distance (Petersik's Figure 3) shows nearly linear declines in rotation discrimination with successively greater simulated projection distances for three different ISIs. On the other hand, the comparable function for perceived depth shows sharp declines only at short projection distances $(1.5,3.0$, and 5.0 sphere radii) and subsequently asymptote. The difference between the two sets of functions can be construed as evidence that depth judgments and rotation judgments are at least partially dissociated.

(3) The correlation between the percentage of correct rotation-direction judgments and mean depth ratings across all conditions of both of Petersik's experiments was determined for each subject. In Experiment 1 , three of the eight subjects had significant positive correlations between the two judgments, rs $=.504, .386$, and $.699(\mathrm{p}<.01)$. These results do not seem to warrant the conclusion that there is necessarily a dependency between the two types of perceptual judgment, although for some subjects that would certainly appear to be the case. It remains to be answered why some, but not all, of the subjects produced such high correlations.

(4) Difference in perceived depth between correctly and incorrectly perceived directions of rotation: As another piece of evidence on the issue of whether depth and rotation-direction judgments are mediated by dissociated perceptual processes, the depth ratings corresponding to spheres whose rotation direction was correctly judged were compared with the depth ratings for spheres whose rotation direction was incorrectly judged. For each subject in Experiment 1, separate records were kept for depth judgments that were provided both when rotation judgments were correct and when they were incorrect. The mean depth rating for each subject in each condition was next determined for both the correct and incorrect rotation judgments. In some conditions, some subjects made no incorrect rotation judgments and thus had no corresponding mean depth judgments. In such cases, the mean depth rating assigned to the "incorrect" category was the same as the mean depth rating that was obtained for the correct judgments. Notice that this rule would tend to favor the null hypothesis that no difference exists between the depth ratings assigned to spheres whose rotations are correctly and incorrectly judged.

Figure 1 shows, for both the spheres whose rotation directions were correctly and incorrectly judged, the mean depth ratings as a function of ISI. As can be seen, at each ISI the mean depth rating for the correct rotation judgments is greater than the corresponding depth rating for the incorrect rotation judgments. The mean depth ratings were entered into a two-way repeated measures analysis of variance with ISI and correctness of rotation judgments as factors. As was found in a previous analysis, there was a significant effect of ISI $(\mathrm{p}<.001)$. In addition, there was a significant main effect of correctness of rotation judgments on associated depth judgments $[F(1,7)=$ $7.70 ; \mathrm{p}<.05 ; \mathrm{SE}=.06]$.

The finding that the magnitude of perceived depth depends upon the correctness of perceived rotation direction implies that the two judgments cannot be completely dissociated. In view of this result, the conclusion that, at some level of processing, the same perceptual process(es) influences depth judgments as well as rotation-direction judgments seems to be inescapable. One reason why earlier attempts to assess the dependency between the two judgments may have failed is that rotation direction is not a prothetic, but rather a metathetic, sensation. That is, in these experiments there were not degrees of rotation direction

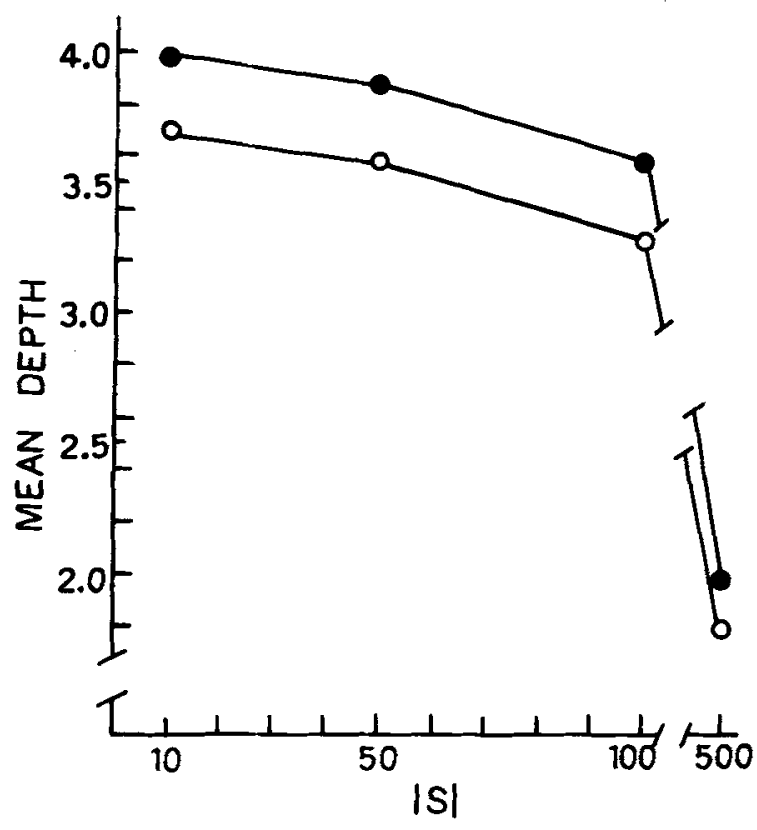

Figure 1. The relationship between correctness/incorrectness of rotation-direction judgments and mean depth ratings. Depth ratings are shown as a function of ISI (in milliseconds). es: correct rotation-direction judgments; Os: incorrect rotation-direction judgments. 
like there were degrees of perceived depth. There were only two states of rotation direction, clockwise and counterclockwise. As Stevens (1975) has shown, prothetic and metathetic sensations give rise to psychophysical scales with different properties, and direct comparisons between them are not fruitful. Therefore, according to this point of view, it is not until we examine the prothetic sensations (e.g., depth) as a function of the state of a related metathetic sensation (e.g., rotation direction) that dependencies can be revealed.

However, the problem remains as to why there should be a dependency of any kind between the two types of perceptual judgment. To claim that incorrect rotation-direction percepts give rise to smaller depth judgments assumes that the visual system can, at some level, determine the difference between its own correctness and incorrectness. Although such a possibility is not impossible, it does seem to be unrealistic. Two other possibilities exist, however. First, rotationdirection judgments might depend on the perception of some minimum amount of depth in the rotating sphere. The argument is that if subjects do not perceive much depth in a sphere, they would probably also have difficulty discriminating the front and rear surfaces of the sphere and thus would also have difficulty in judging the rotation direction of the sphere (since the points in the front and rear hemispheres travel in opposite directions during rotation). Secondly, the processes underlying the depth sensations and the rotationdirection sensations might have just a few mutual connections or lateral interactions. Thus, only certain causes of inefficiency (i.e., those that affect common connections) in one process would simultaneously affect other processes. It is not easy to decide exactly between these latter two alternatives. If rotation direction did depend upon perceived depth, as the first alternative implies, then any factor that reduces perceived depth should also reduce the percentage of correct rotation-direction judgments. As shown below, however, different patterns of variables seemed to have influenced the two judgments in Experiments 1 and 2. Thus, the first alternative seems unlikely. On the other hand, the second alternative seems to be less vulnerable to the same criticism, since it assumes that not all of the same variables need to affect both the depth and rotation-direction processes. Therefore, at present, the second of the two alternatives mentioned above seems to be more likely to account for the observed dependency between the correctness of rotation judgments and perceived depth.

(5) Finally, and perhaps least conclusively, there was not much commonality between the rotationdirection judgments and depth judgments in terms of the patterns of variables that influenced them. In fact, of the nine simple main effects and interactions that we intended to examine, only three (ISI, simulated projection distance, and SOA) had significant effects on both dependent measures. A lack of correspondence between the effects of independent variables on depth and direction judgments, such as that found here, led Braunstein (1976) to his original conclusion that depth and direction judgments are functionally dissociated.

Contrary to the results of Braunstein (1962), in the present experiment dot numerosity $(5,10,25$, or 60 dots per sphere) had no main effect on either dependent measure.

\section{Conclusions}

Most of the evidence considered thus far suggests that we may reject the null hypothesis of no difference between the processes underlying rotation discrimination and depth judgments. Nonetheless, we should not hasten to accept Braunstein's alternative hypothesis that the two judgments rely upon "separate processes." Our caution is demanded by the demonstration of some high individual correlations between rotation-judgment accuracy and perceived depth and by the apparent dependency of depth judgments upon rotation-discrimination accuracy (or vice versa). The strongest conclusion we can draw at this time is that the two judgments are not completely independent. The nature of the relationship between them awaits further experimentation.

\section{REFERENCE NOTE}

1. Pantle, A. J. Research on visual perception of complex and dynamic imagery (Report No. AMRL-TR-77-83). Wright-Patterson Air Force Base, Ohio: Aerospace Medical Research Laboratory, Aerospace Medical Division, Air Force Systems Command, November 1977.

\section{REFERENCES}

Braunstein, M. L. Depth perception in rotating dot patterns; effects of numerosity and perspective. Journal of Experimental Psychology, 1962, 64, 415-420.

Braunste in, M. L. Depth perception through motion. New York: Academic Press, 1976.

Petersik, J. T. The effects of spatial and temporal factors on the perception of stroboscopic rotation stimulations. Perception, in press.

Richards, W. Visual space perception. In E. C. Carterette \& M. P. Friedman (Eds.), Handbook of perception (Vol. 5) Seeing. New York: Academic Press, 1975.

Stevens, S. S. Psychophysics. New York: Wiley, 1975.

\section{NOTE}

1. I thank John Jahnke of Miami University for suggesting the term "functional dissociation." We believe that it is less theoretically loaded than the term "independent."

(Received for publication March 3, 1980; accepted March 10, 1980.) 